\title{
Digital Library Technology for Locating and Accessing Scientific Data
}

\author{
Robert E. McGrath, Joe Futrelle, Ray Plante, \\ National Center for Supercomputing Applications \\ University of Illinois, Urbana-Champaign \\ Damien Guillaume, \\ Université Louis-Pasteur, Strasbourg, France
}

\begin{abstract}
In this paper we describe our efforts to bring scientific data into the digital library. This has required extension of the standard WWW, and also the extension of metadata standards far beyond the Dublin Core. Our system demonstrates this technology for real scientific data from astronomy.
\end{abstract}

KEYWORDS: Information Retrieval, Scientific Data, Astronomy Data, Scientific Information Systems

\section{INTRODUCTION}

In the last few years we have seen the evolution of the Internet and WWW from a loose connection of information sources toward information rich digital libraries. (e.g., [2, 3, 12, 11]) In our view, a digital library is an organized way to locate, access, and analyze digital artifacts of many kinds, federating many data services to create a virtual information space.

The evolving digital library may play a key role for scientists by providing a unified environment for information discovery and access. In particular, the digital library can go beyond the traditional library, and provide direct, immediate location and access to both literature and data.

At NCSA, the Emerge project has been constructing the basic infrastructure required for interoperable searching and for analysis of many kinds of data from many heterogeneous data services distributed about the network. 18] In our current work, we are implementing a prototype to demonstrate the effectiveness of this technology for searching and accessing astronomy data. This prototype illustrates how our flexible architectures can be applied to a collection of existing systems, to create an enhanced environment for information discovery. [21] This work has been a collaboration of astronomers and computer scientists and NCSA, NASA, University of Ulster, and elsewhere. The NCSA Astronomy Digital Image Library (ADIL) has been the key testbed for demonstrating the technology. [24, 25]

In this paper, we describe our model and prototype implementation for interoperable search and analysis as applied to scientific digital libraries. Our model places constraints on standards necessary for meaningful interoperability. In particular, we will show that the Dublin Core alone is insufficient to support the metadata associated with complex scientific data. However, appropriate standards can facilitate richer forms of research tuned to a distributed scientific environment.

\section{Digital Libraries for Science}

For scientists, the digital library is particularly important because of the importance of digital data and analysis, and the need for timely and rich information exchange. Today, scientific discovery and communication routinely creates and uses many kinds of significant digital components:

- raw data

- analyzed data

- imagery

- analysis environments

- simulations 
- notes, letters, and reports

- published articles

These digital artifacts are complex and inter-related; for example, a digitally published article "points" to the data, instrumentation, and software which are described and interpreted by the text. Similarly, the digital representations of simulations and analyzed data such as images are most useful and valid in the context of the published documentation and scientific reports. An archive of scientific data will contain pointers from the data to published articles which explain and validate them; and scientific articles contain pointers to the data which they report. Similarly, theoretical results in the form of computational models are intended to be correlated with relevant observational and experimental data.

There are many repositories of scientific data already on line, and each new scientific project almost inevitably produces significantly larger amounts of digital data. 32] Through the use of the World Wide Web and URLs, scientific information is already becoming a rich web of connected digital information. However, it remains a significant and lasting challenge for humans to exploit this richness, to discover, access, and understand the knowledge that may reside or be created from digital resources. We believe that these archives should be an integral part of the digital library of the future, bringing together all types of scientific information resources in a single environment.

The Emerge project at NCSA is developing practical infrastructure for this new type of digital library. In this vision, a student or researcher could "go to the library" to ask a scientific research question. For example, a researcher could seek to inquire about the climate in Illinois in recent years. Even today, the library would provide pointers to published literature about weather, vegetation, wildlife, and so on, much of which is available on-line. The results should also provide pointers to relevant climate data, satellite imagery, computational models, and resources such as email archives. In most cases, these resources already exist and are available on the Web, but locating and accessing this diverse set of materials would be difficult without the organizing and facilitating role of a new kind of research library. This kind of digital library will not only make routine scientific information finding more efficient, it will enable cross-discipline and synergistic discovery; since the investigator will likely be presented with information from many unexpected sources.

\section{A Case Study: Astronomy and Space Science Data}

In recent decades, we have experienced a golden age for the exploration of the universe. New ground and space based instruments and powerful computing systems have produced an explosion of astronomy and space science data. This explosion has driven the development of data archives, digital libraries, and other network-based services that make it easier to access research-quality information. The success of such services has created environments within which one can gather knowledge from diverse sources to address new scientific questions.

\section{The Astronomy Digital Image Library (ADIL) Testbed}

The NCSA Astronomy Digital Image Library

(ADIL) has been the key testbed for demonstrating the technology. The ADIL was developed with support from NASA and the National Science Foundation to address some of the challenges of distributing scientific data over the network. [24, 25] Its specific mission is to collect fully processed astronomical images in FITS format (a standard astronomical image format [15]) and make them available to the research community and the interested public via the World Wide Web.

The ADIL allows users to search, browse, and download astronomical images. As we will discuss below, this can be a non-trivial process when the images are not in the usual GIF or JPEG formats.

The ADIL is more than a tool for astronomers looking for images to augment their research. It is also a means for authors who wish to share their images with the community. While many of the Library's images come from observatories, the core of the collection comes from individual authors. The ADIL provides a way to upload the images to the Library, along with any supporting data, where it can be processed and made available to the Library users.

Authors deposit images into the Library in the form of collections we refer to as "projects". Normally, 
an author would make a deposit at the end of some scientific study when the resulting publication is going to press; all the fully processed images associated with that paper would make up the project. In this way, the ADIL is part of the new paradigm for scientific publishing. 24, 25]

\section{EXTENDING THE WWW MODEL: A 'CONVERSATION WITH THE DATA'}

In the conventional WWW model, which is biased toward small, text-oriented documents, a data location service usually returns a set of URLs pointing to documents which the user must visit-i.e. download to the client-to view and analyze. For scientific data, "search-and-download" is not a practical model because the objects are typically not "documents", but rather large, complex objects (datasets) stored in formats not supported by standard browsers (such as FITS [15] or HDF [19]). In earlier work, we described the need for a "conversation with the data" which extends the standard Web model. [4, 13. The basic scenario is:

1. search to locate candidate data objects

2. browse and select the objects

3. download selected data for further analysis

Scientific archives have adapted to the Web by integrating a browsing stage to the information discovery process (e.g. [24, 33]). In so-called server-side browsing, the data provider presents a preview of a dataset which might include a GIF or JPEG rendering of the data and a display of some subset of the associated metadata, all packaged as an HTML document. The ADIL is a good example of such a data service.[1]

This model can work very well when interacting with a single data provider and a set of datasets that isn't too large. However this model becomes quite laborious to the user when trying to interact with more than a few weterogeneouwith s data providers because:

1. a single question must be entered differently into each of the providers' custom interfaces
2. each HTML-formatted query response and associated browsing documents must be visited for visual interpretation in a series of individual, stateless requests, e.g., a list of links to URLs.

3. browsing of the data items is limited to what is provided by each data providers' interfaces.

Also, some kinds of user interaction are difficult to implement with server-side browsing. For instance, drawing a bounding box or dynamically fiddling with color maps is difficult to implement well on a server.

To address these issues we extend the "search-browseand-download" model by adding:

1. stateful communication with a data-provider,

2. support for standard query profiles,

3. support for standard record format appropriate for scientific data.

The result is a more fluid, automated, and efficient interaction with multiple data providers. Users can interact with data from one provider while queries to other providers are being processed. The browsing can occur with different levels of detail. Perhaps the most powerful feature is that clients can take greater control of the browsing by plugging in specialized visualizers for quick plotting or manipulating of the results.

\section{INFRASTRUCTURE FOR INFORMATION DISCOVERY FOR SCIENTIFIC RESEARCH}

Information discovery is increasingly the most critical component of scientific research. As scientists work to solve problems, they need multi-modal access to geographically-distributed collections of large and highly structured data sets. Discovering which data sets are potentially relevant to a particular problem involves more or less elaborate characterizations of the data in terms of domain-specific attributes. Furthermore, examining candidate data sets to locate the most relevant ones involves highly specialized interactions with the data.

Yet this diversity must not come at the cost of interoperability. For science to progress, it is crucial that scientists be able to locate information 
from many different scientific domains when attempting to solve a problem in their own domain. Cross-disciplinary researchers should not be burdened with a different set of information discovery software tools for each discipline they work in, especially in the respects that those software tools perform essentially the same functions. Also, data services should provide data not only to individual end users but also to other services which add value to them.

Even within a single discipline, it may be necessary to query many data repositories in order to locate all the data relevant to a scientific question. For instance, the NASA Space Science Data System Technical Working Group reports a real scenario based on the investigation of sulfur $\left(S_{2}\right)$ on comets. This investigation turned out to require data from multiple sources, including several spacecraft, ground based telescopes, the Hubble Space telescope, and published (and unpublished) scientific literature. The data was retrieved from many different sources, in widely different formats. ([16], Appendix 2)

A distributed information discovery infrastructure should be built which emphasizes standard search protocols, file formats and general purpose-tools. It should be designed in such a way that profiles, formats, and browsers specific to a particular domain can be easily plugged in to the infrastructure and shared between data providers and consumers.

In a sense, the WWW already provides a semblance of such an infrastructure. HTTP supports a variety of file formats and forms-based CGI services can be used to implement search tools which return views of information with additional forms controls for manipulating the view. However this mode of using the WWW does little to advance a standard query syntax or means of defining metadata schemas and profiles. Also, it fails to separate the user interface for information retrieval (the HTML forms) from the delivery of information itself (the metadata in the page). Search results returned as part of an HTML page are not standardized and cannot be easily be compared to similar results from a different service.

\section{NCSA Emerge: Practical Infrastructure For Information Discovery}

The NCSA Emerge Project is addressing these issues, with the goal of designing infrastructure to create unified information discovery across heterogeneous databases; developing free software based on standards (Z39.50 [34], XML 30]). The Emerge software includes [18]:

- the Gazelle gateway, which adds Z39.50 to a database (using Z39.50 is recommended but not required)

- the Gazebo search gateway, which manages searches across multiple heterogeneous databases

- a Java client toolkit, which communicates with the Gazebo gateway, creating queries and presenting results.

This infrastructure is being developed for several applications, including engineering literature [3] and medical research databases, as well as astronomy.

\section{Profiles}

In order to build a distributed search infrastructure which serves the scientific community, we see the need for the following requirements:

- Profiles which are extensible to particular domains

- Protocols for remote access to data collections

- Query syntax and semantics for searching data collections

- File formats for data sets or subsets (e.g., XML document types)

- Flexible record formats for metadata describing data items (e.g., XML document types)

In order to search across such a diversity of sources with a single query, there must be some sort of "common denominator", a common set of search terms with shared semantics. The Dublin Core and related W3C efforts provide this kind of standard for many kinds of "document-like objects". 23, 28]

However, scientific data require metadata considerably beyond the Dublin Core. For example, in addition to the Dublin Core categories, the ADIL supports searches by:

- Sky position (e.g., in galactic coordinates)

- Astronomical Object (e.g., M31)

- Type of object (e.g., galaxy)

- Wavelength 
Still other types of metadata are needed for archives of planetary data, such as orbital positions and descriptions of atmospheres and clouds. While each discipline-and maybe even each project and instrumentmay require some unique metadata, we believe there is enough common ground to establish standard profiles for broad classes of scientific data, just as the Dublin Core has done for documents. (See our proposals for astronomy metadata in [14, 26].)

There must also be standards for the format of the results of queries for data; a structured record that describes the kinds of objects returned as data, such as images, tables, and datasets in various formats such as FITS. USMARC records have served this role for many years for bibliographic material, but new, more flexible record formats are needed to support scientific data. The W3C RDF and schema initiative 29 provides a sound framework for expressing such records, but it is critical for communities to work to establish the appropriate standards.

\section{AML: Astronomical Markup Language, a metadata stan- dard for astronomy}

The Astronomical Markup Language (AML) addresses the needs for standardized metadata for Astronomy data. AML is an XML language describing various kinds of data useful in astronomy, and is aimed at being an exchange format for astronomical data, and especially metadata, over the Internet. AML is both a proposed profile standard and a prototype implementation. [7, 8]

Results of a search can be formatted as an AML document, that is, as an XML document containing a description of the resource using the AML DTD. The AML document can be processed by a program or presented by a browser. Guillaume has created a Java applet to browse AML documents as easily as one would browse HTML documents, but with some additional features specific for astronomical data. For example, the AML applet displays astronomical coordinates, and displays measurements with the relevant units and uncertainties. [7, 8]

The use of AML is an improvement for both the information providers and the users (who are astronomers). For the information providers, XML separates the data from the user interface, so that different data can be used with different user inter- faces without any difficulty. A small institute could also focus on the information, and let other institutes provide user interfaces. For the users, the use of the AML browser provides a uniform and unified way to access various data coming from different servers. Finally, users wanting to get and process the data automatically, can use the AML documents directly, as the AML browser applet does. $\mathrm{XML}$ is much more useful for this purpose than HTML, because HTML documents contain a mix of information about both the user interface and the data.

The AML language is organized as seven types of objects. An AML document is a collection of AML objects, describing different types of information. AML objects may contain links to other AML objects, and to external objects such as data, images, or documents. The AML objects are summarized in Table 1. The AML language can be easily extended, for example by adding a "Set of images" object.

AML records are designed to allow programs to automatically process and analyze the metadata. Guillaume has demonstrated techniques for automatically clustering astronomical information sources, e.g., applying a graph partitioning algorithm to the keywords and links in AML records. 99 One outstanding feature of this work was that information from diverse sources was successfully correlated, because the AML records are standardized. It is easy to imagine how this work could be extended to support filtering for specific users and selective dissemination of information.

\section{INTERACTION WITH THE DATA}

The search system described above locates information sources, and returns AML records to describe them. From the AML records, the user identifies data that appears to be of interest. There may in fact be a large number of large datasets, so it is important for the user to select subsets and subsamples from the data. For instance, it may be the case that only one region or time period is required, or only certain measurements are relevant.

Sometimes the metadata itself is not sufficient to make the selection, in which case the user needs to browse data itself. A low resolution "thumbnail" 
Table 1: The objects defined by the Astronomical Markup Language

\begin{tabular}{|l|l|}
\hline Object & Definition \\
\hline Metadata & $\begin{array}{l}\text { An AML document is usually composed of the metadata part, } \\
\text { and of one the other parts. }\end{array}$ \\
\hline Astronomical object & $\begin{array}{l}\text { This describes information about an astronomical object, } \\
\text { with the identifiers (the names), the coordinates, the object } \\
\text { type, other information, a list of measurements }\end{array}$ \\
\hline Article & $\begin{array}{l}\text { This part only describes information about an article, includ- } \\
\text { ing links to the article, if available. }\end{array}$ \\
\hline Table & Metadata for a table, and a link to the content of the table. \\
\hline Set of tables (catalogue) & $\begin{array}{l}\text { A set of tables is a list of tables linked together, with infor- } \\
\text { mation about the set. }\end{array}$ \\
\hline Image & $\begin{array}{l}\text { Metadata about an image and the way it is stored, and a link } \\
\text { to the raw data of the image. }\end{array}$ \\
\hline Person & $\begin{array}{l}\text { Information about a person, usually an author of astronomi- } \\
\text { cal articles. }\end{array}$ \\
\hline
\end{tabular}

image may be viewed, and the user may ask to pan and zoom around the image, to examine in detail areas of possible interest. Regions of an image may be selected with a bounding box, data from tables may be examined, from which particular columns (fields) and rows selected. It may be useful to make simple histograms or other plots, to identify characteristics of the data, and it may be useful to manipulate the color tables or other aspects of the display to highlight features of the imagery. The dataset may contain tables of data, or other multidimensional data structures, all of which need to be efficiently navigated in a similar fashion.

When the precise data of interest is identified, the user then requests subsets and subsamples to be downloaded for detailed analysis. At this point, the data will be input to data analysis programs or simulations. These may range from simple graphing and spreadsheets, up to complex, multi-supercomputer environments. In any case, the results may ultimately be published, adding new documents and datasets to the library.

In the case of the ADIL, the FITS data might be filtered, combined with data and models, and visualized. This might be done using AIPS ++ or a similar package. 22] The results of the analysis would be saved as one or more FITS images, which might be entered in the ADIL when the study is published in a journal. [24, 25]
Because of the elaborateness of scientific data, even the retrieval step itself can sometimes involve fairly complex calculations above and beyond the boolean matching typical of bibliographic data; e.g., applying a pattern recognition algorithm to a database of images. Furthermore, data may need to be processed and formatted even before it's browsed. And finally, the scientific investigation may involve analysis of multiple data sets to produce a composite data product distilled from diverse data from several sources. Today, these types of activities are carried out routinely using a heterogeneous, adhoc assortment of applications, typically specialized applications requiring access to data on local disks. In the future, this will increasingly be done using "workbenches" (i.e., specialized Web portals such as [17) and ubiquitous computational GRIDs. [6, 5

\section{A PROTOTYPE IMPLEMENTATION}

Over the past few years NCSA Project 30 has been constructing a prototype which provides a sophisticated "conversation with the data" for astronomy data. Our prototype uses NCSA Emerge and AML as the basis to build a system to locate, browse, and retrieve astronomy data from the NCSA Astronomy Digital Image Library and other data services.

The data sources are already available through standard Web interfaces which return HTML. We have added the ability to use Z39.50 to query, installing 
the Gazelle Z39.50 gateway on the data server if needed.

The Gazebo GUI implements a query construction interface, which presents one or more profiles, i.e., standard sets of query terms and meanings. The client configuration is loaded from the Gazebo gateway, so the same client can have many "views" of the information space. The current prototype implements both a "simple" query interface (a single list of keywords), and an "advanced" interface (a graphical interface to construct a boolean expression). The prototype supports a general purpose profile for bibliographic searching, and a specialized astronomy profile.

The results of the query are returned as AML records, as well as HTML. Creating AML (XML) records is usually a straightforward extension of the existing code that generates HTML.

The Gazebo GUI sends queries, encoded in the XML-based Gazebo abstract search language, to the Gazebo gateway to be executed on a set of target data sources. Gazebo translates each query into the native query syntax of each target data source, and executes it remotely using the native search protocol of each target data source. This behavior is highly configurable. Requesting result records is handled similarly; Gazebo translates the GUI's requests for results into the target data sources' protocols.

The result records returned by typical data sources are more or less structured data. Gazebo can return them unmodified or it can process them through external CGI scripts which can translate them from arbitrary file formats into any MIME type. This is useful, for instance, for providing HTML views of records in non-text formats. The records are passed from the gateway to the GUI, which displays them appropriately.

The GUI displays the number of records returned by each server, and retrieves and shows the short records as requested. When a full record is requested, the GUI retrieves the record and launches an appropriate applet to display it. If the record is HTML, it is displayed with the ICE HTML viewer. [10] When the record is AML, the AML browser is invoked to display it.
The AML record may contain pointers to abstracts and/or datasets. The user may follow these links to view the actual data. The abstract will be viewed with the HTML viewer. When a FITS dataset is selected, the Horizon Image Browser [20] will be launched to browse the data, and download it if desired.

\section{CONCLUSION AND RELATION TO OTHER WORK}

We have constructed a complete environment for locating astronomy information, for examining and browsing metadata, and for browsing and accessing both the text and the data. Our system is unique in that we support both text and data, using a general, standards based protocol. We have defined new protocols for describing astronomy data, and created a much more complex "conversation" than most systems can support. The flexible configuration and interoperable standards we use make it comparatively easy to add databases.

It is important to reiterate that the Emerge software is extremely flexible, and is used for several application communities. The astronomy specific features are replaceable modules, the system can be customized for different user communities.

The Gazebo gateway superficially resembles many conventional Web gateways and portals. However, we use Z39.50 to distribute the queries and AML to return the results. These standards assure much greater interoperability than Web CGI and HTML.

Z39.50 has been widely used by libraries for many years, and there are many efforts to federate Z39.50 services, such as the CIC Virtual Electronic Library. [2] There is also a well established effort to standardized metadata for bibliographic resources, e.g., the Dublin Core. 223] Our work is important because it shows that Z39.50 can be used with scientific data. Our protocol development and the AML extend the principles of the Dublin Core to a significant body of scientific data.

The AML uses standard XML, but is not directly related to the still evolving W3C metadata efforts. [28, 29] As RDF standards become established, AML can presumably be aligned with them. For instance, the XML RDF schema [29] and the XML-Data proposal [31] are likely to be important, and the AML will follow these standards as they become estab- 
lished.

The Gazebo gateway and GUI implement a Query protocol using XML. The W3C is currently in the early stages of defining a standard for representing queries in XML. 27] When this standard matures, Gazebo will support it appropriately.

\section{ACKNOWLEDGEMENTS}

This work was partly funded by NASA Office of Space Science [21], the National Science Foundation, and the National Center for Supercomputing Applications (NCSA) at the University of Illinois, Urbana-Champaign. NCSA is funded in part by the National Science Foundation, the Advance Research Projects Agency, corporate partners and the state and University of Illinois. Earlier parts of this work were funded by Project Horizon, a NASA cooperative agreement. Some parts of this work were funded by the NSF/DARPA/NSF Digital Library Initiative, and by the National Cancer Institute.

\section{REFERENCES}

1. Anderson, M., Rudnick, L., Leppik, P., Perley, R. and Braun, R., "Relativistic electron populations in Cassiopeia A", ADIL, 1995, http://imagelib.ncsa.uiuc.edu/document/95.MA.01.01

2. CIC Center for Library Initiatives, "CIC Virtual Electronic Library", http://www.cic.uiuc.edu/cli/accessvel.htm

3. DLI, "The DLI Testbeds: Today and Tomorrow", D-Lib Magazine, July 1996, http://www.dlib.org/dlib/july96/07contents.html

4. Folk, M. and McGrath, R. E., "Project Horizon: Problems addressed, lessons learned and accomplishments", Presentation to Federal Webmasters Workshop, August 7, 1996. http://hdf.ncsa.uiuc.edu/horizon/Webmaster.7.Aug.96/

5. Foster, I. and Kesselman. C., eds., The Grid: Blueprint for a New Computing Infrastructure, Morgan Kaufmann, 1998.

6. globus.org, "The Globus project", http://www.globus.org

7. Guillaume, D., "AML page", http://www.infm.ulst.ac.uk/ damien/these/
8. Guillaume, D. and Murtagh, F., "Clustering for Knowledge Discovery in Astronomy based on Astronomical Markup Language (AML) Documents", ADASS VIII, Urbana, IL, November, 1998.

http://monet.astro.uiuc.edu/adass98/talks/node42.html\#619

9. Guillaume, D., "An application of XML and XLink using a graph-partitioning method and a density map for information retrieval and knowledge discovery",

http://www.infm.ulst.ac.uk/ damien/these/AML_maps.htm

10. ICEsoft S/A, "Welcome to ICEsoft", http://www.icesoft.no/

11. Lagoze, C. and Fielding, D., "Defining Collections in Distributed Digital Libraries", D-Lib Magazine, November, 1998, http://www.dlib.org/dlib/november98/lagoze/11lagoze.htm

12. Leiner, B. M. "The NCSTRL Approach to Open Architecture for the Confederated Digital Library", D-Lib Magazine, December, 1998, http://www.dlib.org

/dlib/december98/leiner/12leiner.html

13. McGrath, R. E., "A Scientific Data Server: The Conceptual Design",

http://hdf.ncsa.uiuc.edu/horizon/DataServer/sds_design.htm

14. McGrath, R., Plante, R., Futrelle, J. and Plutchak, J., "Astro-1 Attribute Set", draft of Z39.50 binding for Astronomy data profile, http://webstar.ncsa.uiuc.edu/Project30/Profile/AstroProfile.htm,

15. NASA, "FITS Support Office Home Page", http://fits.gsfc.nasa.gov/

16. NASA Space Science Data System Technical Working Group, "The NASA Space Science Data System: A White Paper", July 21, 1998, http://ssds.nasa.gov/white_paper.htm

17. NCSA, "Biology WorkBench", http://biology.ncsa.uiuc.edu 
18. NCSA, "NCSA http://emerge.ncsa.uiuc.edu

19. NCSA, "HDF http://hdf.ncsa.uiuc.edu

20. NCSA, "The Horizon Image Data Browser Package", http://imagelib.ncsa.uiuc.edu/imagelib/andJava.html\#horizon.

21. NCSA, "Project 30 Home page", http://webstar.ncsa.uiuc.edu/Project30/

22. NCSA Radio Astronomy Image Group, "Astronomical Data Reduction on Parallel Computers Using AIPS++", http://monet.astro.uiuc.edu/aips++/index.htm

23. OCLC, "The Dublin Core: A Simple Content Description for Electronic Resources", http://purl.oclc.org/dc/Model

24. Plante, R. L., "The NCSA Astronomy Digital Image Library: The Challenges of the Scientific Data Library", D-Lib Magazine, October 1997 , http://www.dlib.org/dlib/october97/adil/10plante.htm
29. W3C, "Resource Description Framework (RDF) Schema Specification", W3C Working Draft 30 October 1998, http://www.w3.org/TR/WD-rdf-schema/

30. W3C, "Extensible Markup Language (XML(TM))", http://www.w3.org/XML/

1. W3C, "XML-Data", http://www.w3c.org/TR/1998/NOTE-XML-data

32. Williams, R., "Workshop on Interfaces to Scientific Data Archives: Report", Pasadena, CA, 24 March 1998. http://www.cacr.caltech.edu/isda/report.htm

33. Yeager, N., "A Web Based Scientific Data Access Service: The Central Component of a Lightweight Data Archive", International Society for Photogrammetry and Remote Sensing (ISPRS) Joint Workshop, October 7-9, 1997 Boulder, Colorado. http://hopi.ncsa.uiuc.edu/ogis/isprspaper.htm

34. Z39.50 Maintenance Organization, "Welcome to the Library of Congress Maintenance Agency page for International Standard Z39.50", http://www.loc.gov/z3950/agency/

25. Plante, R., Crutcher, R., and McGrath, R., "The NCSA Astronomy Digital Image Library: From Data Archiving to Data Publishing", for Future Generation Computer Systems (Elsevier), submitted, 1998.

26. Plante, R., McGrath, R., and Futrelle, J., "A Model for Cross-Database Searching of Distributed Astronomical Information Resources", http://monet.astro.uiuc.edu/ rplante/topics/P30/sysmodel.html

27. Vanderbilt, P., "Query languages for scientific data", Position paper for W3C QL'98 workshop. 1998.

http://www.w3c.org/TandS/QL/QL98/pp/queryreq.htm

28. W3C, "Metadata Activity Statement", http://www.w3.org/Metadata/Activity.htm 